\title{
Session Aware Music Recommendation System with User-based and Item-based Collaborative Filtering Method
}

\author{
M. Sunitha ${ }^{1}$ \\ T. Adilakshmi², PhD \\ ${ }^{1,2}$ Dept. of CSE \\ Vasavi College of Engineering \\ Ibrahimbagh, Hyderabad-31, AP, India.
}

\begin{abstract}
-
Recommender systems have been proven to be valuable means for web online users to cope with the information overload and have become one of the most powerful and popular tools in electronic commerce. The recommendations provided are aimed at supporting their users in various decision making process, such as what items to buy. In Music Recommendation Syste m, we recommend items to users based on their interest. First we use collaborative filtering method to identify the items which are similar and similarity among users based on the users listening history. Proposed Algorithm recommend the items to new users based on the item clusters and user clusters formed. Later we have taken timestamp of user logs also into consideration to form Sessions. Finally we have evaluated the performance of the proposed algorithm with sessions and with -out sessions. Our experiment show that the accuracy of recommendation system with sessions outperformed the conventional user-based \& item-based collaborative filtering method.
\end{abstract}

Keywords- collaborative filtering, recommender system, Item-based clusters, user-based clusters sessions

\section{INTRODUCTION}

Due to the evaluation of internet and e-commerce users are able to get large volumes of information. This problem is known as Information overloading. It is very difficult to get the information which is interesting to the users. Recommender systems are the one which serves as a good tool for information filtering. Information filtering can be done by using content based filters and Collaborative filters. Content based method depends on the content of the item whereas collaborative filtering is based on user's ratings. $[1,2]$

Music recommender systems are decision support tools that solves the information overload problem by recommending the items that are interesting and relevant to the user, based on the user's music preferences[9]. For example , Last.fm a popular Internet radio and recommender system that recommends songs to users based on their interest and other user's rating on those items. It also allows users to get recommendations based on the artist, album and so on.

Many researchers have proposed different kinds of Collaborative filtering techniques to do quality recommendations to users. CF can be performed based on two different methods. One is User based CF technique and the other is Item based $\mathrm{CF}$ technique $[3,4]$. Both these methods are based on the data structure, User-Item matrix. User based CF technique does the recommendations based on the user's interest and their neighbor's ratings i.e first we will take user interest into consideration and then the neighbor's ratings who are similar to the target user. The basis for this method is if a test user is similar to some user ${ }_{i}$, and user $_{i}$ has rated items $\left\{I_{1}, I_{2}, \ldots ..\right\}$, then recommend those items to the test user.

The main challenges faced by CF techniques are Sparsity, Scalability and Cold-Start [10].

Sparsity: As we compare the number of users with the number of items, a user will rate few items out of total number of available items. Because of this the data structure, User-Item matrix used in $\mathrm{CF}$ techniques will be sparse. Recommendations provided based on these sparse ratings will be less accurate i.e user will be recommended many uninterested items.

Scalability: Scalability is another important problem faced by $\mathrm{CF}$ techniques. The time complexity of $\mathrm{CF}$ techniques increases non linearly with the increase in the number of users or items as they are basically dependent on similarity measures.

Cold-Start: Cold-start is the problem of not able to recommend items to new users and new items to existing users. This is because $\mathrm{CF}$ technique can not recommend items to new users until the user rates sufficient number of items. Similarly CF technique will not be able to recommend new items to users until the items are being rated by sufficient number of users.

This paper uses user listening history for collaborative filtering system based on user clusters and item clusters in music recommendation systems. We also proposed an algorithm for music recommendation by taking Sessions into consideration.

The rest of the paper is organized as follows. Section II deals with traditional collaborative filtering methods. Section III describes about the proposed approach. Section IV explains about the experimental set up and Results. Section V describes about conclusion and future directions for research. 


\section{TRADITIONAL COLLABORATIVE FILTERING ALGORITHMS}

\subsection{User -Item Rating matrix}

The task of the traditional collaborative filtering recommendation algorithm concerns the prediction of the target user's rating for the target item that the user has not given the rating, based on the users' rating on observed items. User-Item matrix is the central of this method. Each user is represented by item-ratings pairs, and can be summarized in a user-item matrix, which contains the Scorings Sij that have been provided by the ith user for the jth item, the matrix as following

\begin{tabular}{|c|c|c|c|c|}
\hline Item /User & Item $_{\mathbf{1}}$ & Item $_{\mathbf{2}}$ & $\cdots \cdots$ & Item $_{\mathbf{n}}$ \\
\hline User $_{1}$ & $\mathrm{~S}_{11}$ & $\mathrm{~S}_{12}$ & $\cdots \cdots$ & $\mathrm{S}_{1 \mathrm{n}}$ \\
\hline User $_{2}$ & $\mathrm{~S}_{21}$ & $\mathrm{~S}_{22}$ & $\ldots \ldots$ & $\mathrm{S}_{2 \mathrm{n}}$ \\
\hline$\ldots \ldots$ & $\ldots \ldots$ & $\ldots \ldots$ & $\cdots \cdots$ & $\cdots \cdots$ \\
\hline User $_{\mathrm{m}}$ & $\mathrm{S}_{\mathrm{m} 1}$ & $\mathrm{~S}_{\mathrm{m} 2}$ & $\cdots \cdots$ & $\mathrm{S}_{\mathrm{mn}}$ \\
\hline
\end{tabular}

Fig. 1 User-Item Matrix

Where Sij denotes the score of item $\mathrm{j}$ rated by an active user $\mathrm{i}$. If user $\mathrm{i}$ has not rated item $\mathrm{j}$, then $\mathrm{Sij}=0$. The symbol $\mathrm{m}$ denotes the total number of users, and $\mathrm{n}$ denotes the total number of items.

\subsection{Similarity Measures}

Collaborative filtering approaches have been popular for both researchers and practitioners alike evidenced by the abundance of publications and actual implementation cases. Although there have been many algorithms, the basic common idea is to calculate similarity among users using some measure to recommend items based on the similarity. The collaborative filtering algorithms that use similarities among users are called user based collaborative filtering [9]

A set of similarity measures are presented and a metric of relevance between two vectors. When the values of these vectors are associated with a user's model then the similarity is called user based similarity, whereas when they are associated with an item's model then it is called item based similarity. The similarity measure can be effectively used to balance the ratings significance in a prediction algorithm and therefore to improve accuracy.

Similarity measures are evaluated as a metric of similarity between two users by using vectors. When the values of these vectors are associated with a user's model then the similarity is called user based similarity. The similarity measure can be effectively used to balance the ratings significance in a Recommendation algorithm to improve accuracy.

The following are the different similarity measures used in user based $\mathrm{CF}$ technique. [1,3] Pearson correlation, cosine vector similarity and adjusted cosine vector similarity etc.
Pearson's correlation, measures the linear correlation between two vectors of ratings.

$$
\operatorname{Sim}(i, j)=\frac{\sum_{c \in j}\left(S_{i, c}-A_{i}\right)\left(S_{i, c}-A_{j}\right)}{\sqrt{\sum_{c \in i}\left(S_{i, c}-A_{i}\right)^{2} \sum_{c \in j}\left(S_{i, c}-A_{i}\right)^{2}}}
$$

Where $S_{i, c}$ is the rating of the item c by user,$A_{i}$ is the average rating of user $i$ for all the co-rated items, and $\mathrm{I}_{\mathrm{ij}}$ is the items set both rating by user ${ }_{i}$ and user $_{j}$.

The cosine is a measure of similarity between two vectors as the cosine of the angle between them. The cosine of $0^{\circ}$ is 1 , and it is less than 1 for any other angle. Two vectors with the same orientation have a Cosine similarity of 1 , two vectors at $90^{\circ}$ have a similarity of 0 , and two vectors diametrically opposed have a similarity of -1 , independent of their magnitude. Cosine similarity is particularly used in positive space, where the outcome is neatly bounded in $[0,1]$.

The cosine of two vectors can be derived by using the Euclidean dot product formula:

$$
\mathrm{a} \cdot \mathrm{b}=\|a\|\|b\| \cos \theta
$$

Given two vectors of attributes, A and B, the cosine similarity, $\cos (\theta)$, is represented using a dot product and magnitude as

$$
\begin{aligned}
\text { Similarity }= & \cos (\theta)=\frac{A \cdot B}{|| A|\||| B\||} \\
= & \frac{\sum_{i=1}^{n} A_{i} X B_{i}}{\sqrt{\sum_{i=1}^{n} A_{i}^{2}} X \sqrt{\sum_{i=1}^{n} B_{i}^{2}}}
\end{aligned}
$$

The adjusted cosine similarity as the formula given below, is used in collaborative filtering methods to find similarity among users when the users ratings are not given the same importance i.e the ratings of different users are taken on a different scale.

$$
=\frac{\operatorname{Sim}(i, j)}{\sqrt{\sum_{c \in j}\left(S_{i, c}-A_{c}\right)\left(S_{i, c}-A_{c}\right)^{2} \sum_{c \in j}\left(S_{i, c}-A_{c}\right)^{2}}}
$$

Where $S_{i, c}$ is the rating of the item c by user ${ }_{i}, A_{c}$ is the average rating of user ${ }_{i}$ for all the co- rated items, and $I_{i, j}$ is the items set both rating by user ${ }_{i}$ and user ${ }_{j}$.

\section{PROPOSED APPROACH FOR RECOMMENDATIONS}

This section describes about the dissimilarity measure used, formation of sessions, formation of user-based clusters and item-based clusters, recommendation of items by taking sessions into consideration to new users and evaluation measures. 


\subsection{Euclidean Distance}

Euclidean distance is a measure of dissimilarity. It measures is the distance between two points by the Pythagorean formula given below.

The distance from $\mathbf{p}$ to $\mathbf{q}$ is given by

$$
\begin{aligned}
& d(p, q)=d(q \cdot p) \\
& =\sqrt{\left(q_{1}-p_{1}\right)^{2}+\left(q_{2}-p_{2}\right)^{2}+\cdots+\left(q_{n}-p_{n}\right)^{2}} \\
& =\sqrt{\sum_{i=1}^{n}\left(q_{i}-p_{i}\right)^{2}}
\end{aligned}
$$

\subsection{Formation of Sessions}

User logs are divided into sessions. A session is defined as fixed time slot of a day. We have taken four sessions for each day of equal intervals i.e from 0 a.m to 6 a.m as $S_{1}$, 6a.m to 12 p.m as $S_{2}, 12$ p.m to 18 p.m as $S_{3}$ and 18 p.m to 24p.m as $\mathrm{S}_{4}$.

\subsection{User-based Clusters}

Each user from the user-item matrix of one of the session $\left(\mathrm{S}_{1}, \mathrm{~S}_{2}, \mathrm{~S}_{3} \mathrm{~S}_{4}\right)$ is considered as a user vector. User clusters for a session are formed by using the following hierarchical agglomerative clustering algorithm

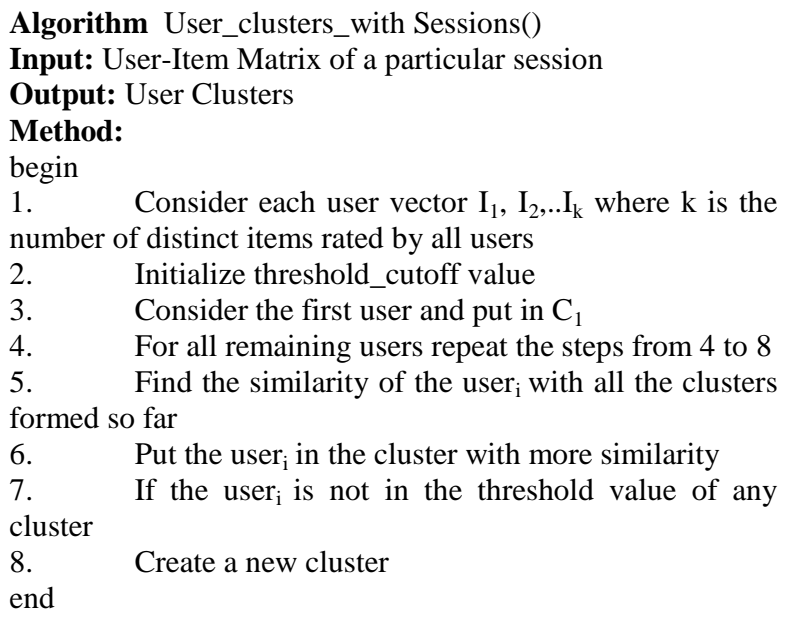

Fig. 2 Pseudocode for User_clusters_with Sessions

\subsection{Item-based Clusters}

Each item from the user-item matrix of one of the session $\left(\mathrm{S}_{1}, \mathrm{~S}_{2}, \mathrm{~S}_{3} \mathrm{~S}_{4}\right)$ is considered as an item vector. Item clusters for a session are formed by using the following hierarchical agglomerative clustering algorithm

\section{AlgorithmItem_Clusters with Sessions() \\ Input: User-Item Matrix of a particular session \\ Output: Item Clusters}

\section{Method:}

\section{begin}

1. Consider each item vector $\mathrm{U}_{1}, \mathrm{U}_{2}, . . \mathrm{U}_{\mathrm{k}}$ where $\mathrm{k}$ is the number of distinct users rated an item

2. Initialize threshold_cutoff value

3. Consider the first item and put in $\mathrm{C}_{1}$
4. For all remaining items repeat the steps from 4 to 8

5. Find the similarity of the item $m_{i}$ with all the clusters formed so far

6. Put the item $\mathrm{m}_{\mathrm{i}}$ in the cluster with more similarity

7. If the item $m_{i}$ is not in the threshold value of any

cluster

8. Create a new cluster

end

Fig. 3 Pseudocode for Item_clusters_with Sessions

\subsection{Recommendation stage}

After getting the user clusters and item clusters for each session, we use these clusters to recommend items to new users by using the following Algorithm for recommendations.

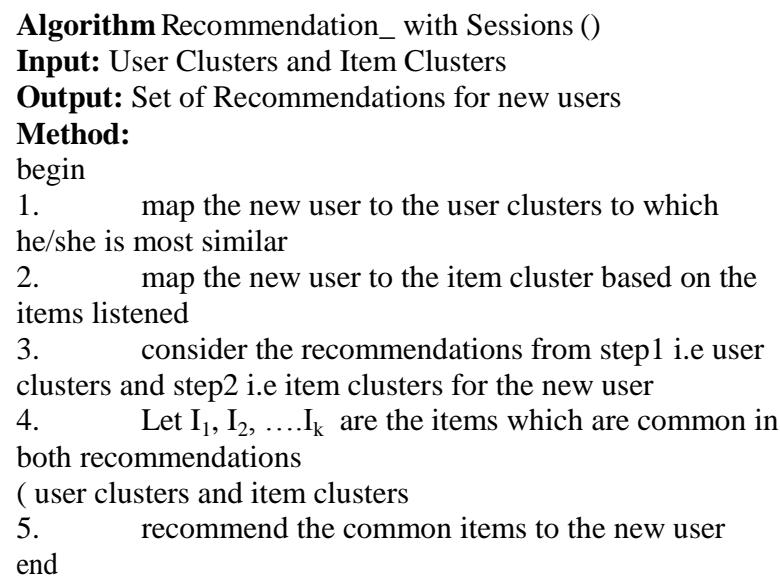

Fig.4.Pseudocode for Recommendation_with Sessions

\subsection{Evaluation Measures}

Evaluating the data mining task is fundamental aspect of machine learning. Many methods have been proposed for assessing the accuracy of collaborative filtering methods. We have used Precision (P) and Recall (R) and $\mathrm{f}$ measure. These measures are obtained from confusion matrix shown in fig. 2

\subsubsection{Confusion Matrix}

A confusion matrix shows the number of correct and incorrect prediction made by the classification model compared to the actual outcomes (target value) in the data.

\begin{tabular}{|c|c|c|}
\hline & Actual-True & Actual-False \\
\hline $\begin{array}{c}\text { Predicted- } \\
\text { True }\end{array}$ & $\begin{array}{c}\text { True Positives } \\
\text { (TP) }\end{array}$ & $\begin{array}{c}\text { False Positives } \\
\text { (FP) }\end{array}$ \\
\hline $\begin{array}{c}\text { Predicted- } \\
\text { False }\end{array}$ & $\begin{array}{c}\text { False Negatives } \\
\text { (FN) }\end{array}$ & $\begin{array}{c}\text { True Negatives } \\
\text { (TN) }\end{array}$ \\
\hline
\end{tabular}

\section{Fig.5 Confusion Matrix}

Precision is a measure of exactness [10], determines the fraction of relevant items retrieved out of all items retrieved. Recall is a measure of completeness, determines the fraction of relevant items retrieved out of all relevant items. Fmeasure is the measure which stabilizes the changes in Precision and Recall. 


$$
\begin{gathered}
\text { Precision }=\frac{\mathrm{TP}}{\mathrm{TP}+\mathrm{FP}} \\
\text { Recall }=\frac{\mathrm{TP}}{\mathrm{TP}+\mathrm{FN}} \\
F-\text { measure }=2 * \frac{(\text { Precision } * \text { Recall })}{(\text { Precision }+ \text { Recall })}
\end{gathered}
$$

\section{EXPERIMENT AND RESULTS}

This section describes about the Dataset used for experiment, experimental set up and results.

\subsection{Data set}

Million Song Dataset (MSD) a freely-available collection of audio features and meta-data for a million contemporary popular music tracks [9]. Comprising several complementary datasets that are linked to the same set of songs. The MSD contains extensive meta-data, audio features, tags on the artist- and song-level, lyrics, cover songs, similar artists, and similar songs. It consists of four datasets namely Last.fm, Second hand data set, Musixmatch and Taste profile data set. We used Last.fm logs as Data set for our experiment

For this experiment, the Last.fm dataset has been used. Last.fm is a music web portal that allows its user base, which has more than 30 million active users, to listen to millions of songs from its music library. All the users' activity is recorded in the Last.fm database, which in turn used by the portal to make music recommendations. The dataset for this experiment contains activities of 48 users whose listening history has been captured anonymously for the period of 3 years. For every song that a user listens to, its activity is recorded in the following format:

User_000004 2009-04-09T12:49:50Z 078a9376$3 \mathrm{c} 0442807 \mathrm{~d} 720 \mathrm{e} 158 \mathrm{f} 345 \mathrm{~d}$

\section{A Perfect Circle}

5ca13249-26da-47bd- bba7-80c2efebe9cd People Are People

\section{Fig. 6 User Record tuple in the dataset}

The above record contains the following fields:

User id (User_000004) - Since the data is captured anonymously, we assigned each

user, a user-id of the format user_000004.

Date-Time (2009-04-09T12:49:50Z) - Time of activity is recorded which will be

used in our algorithm to determine the session in which it will belong.

Album Id (078a9376-3c04-4280-b7d720e158f345d) - A unique identifier is Attributed to each Album.

Album name (A Perfect Circle) - An album to which that song belongs to.

Track id (5ca13249-26da-47bd-bba7-80c2efebe9cd) - A unique identifier is attributed to each track / song.

Track name (People are People) - The songs which the user listened to.

\subsection{Experimental setup}

We have taken 100000 records from Last.fm data set for this experiment. It consists of 48 users listening history for 3 months. We have taken all the items which are listened by at least 2 users. With this constraint on the data set we got 22000 unique items. From 48 users 33 users are taken as training data and 15 users are taken as test data. We formed item clusters by taking $33 \times 22000$ user-item matrix into consideration.

\begin{tabular}{|l|c|c|c|c|}
\hline & Song $_{\mathbf{1}}$ & Song $_{2}$ & $\ldots .$. & $\begin{array}{l}\text { Song } \\
22000\end{array}$ \\
\hline User $_{1}$ & 2 & 0 & $\ldots$ & 0 \\
\hline User $_{2}$ & 0 & 4 & $\ldots$ & 1 \\
\hline$\cdots$ & $\cdots$ & $\cdots$ & $\cdots$ & $\cdots$ \\
\hline User $_{38}$ & 0 & 0 & $\cdots$ & 1 \\
\hline
\end{tabular}

Fig.7. User-Item Matrix for 38 X 22000

\subsection{Results}

We have done the experiment with various values of thresholds such as $0.01,0.02$ and so on till 0.1 without sessions and with sessions.

We plotted the graph for threshold vs Precision for traditional Collaborative filtering and new session based Collaborative filtering. We can conclude from the experimental results that as the threshold value increases the Precision also increases. We can also conclude from the experiment that the Precision of new proposed session aware method has outperformed the traditional method.

\begin{tabular}{|l|l|l|}
\hline $\begin{array}{l}\text { Threshold / } \\
\text { Avg. } \\
\text { Precision }\end{array}$ & with session & without session \\
\hline 0.01 & 0.0355047 & 0.0262619 \\
\hline 0.02 & 0.0549396 & 0.0450402 \\
\hline 0.03 & 0.054887 & 0.0460341 \\
\hline 0.04 & 0.0973042 & 0.0795551 \\
\hline 0.05 & 0.086108 & 0.0606562 \\
\hline 0.06 & 0.1122663 & 0.0707849 \\
\hline 0.07 & 0.1123151 & 0.083704 \\
\hline
\end{tabular}

Table1. Threshold Vs Average Precision

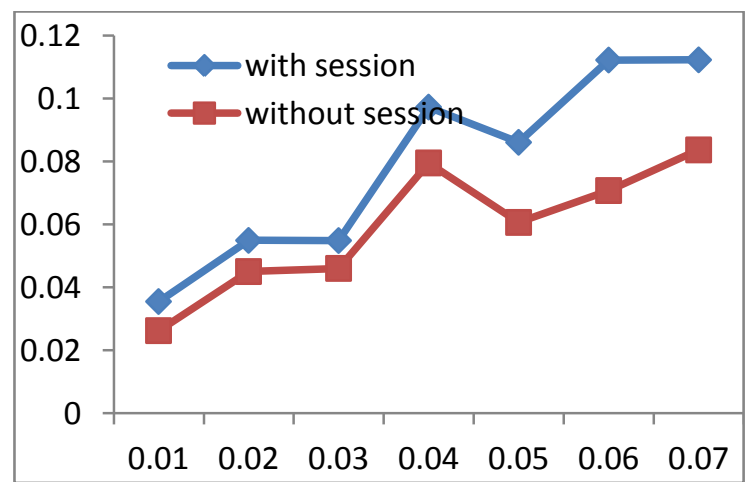

Fig.8 Threshold Vs Average Precision 


\begin{tabular}{|l|l|l|}
\hline $\begin{array}{l}\text { Threshold / Avg. } \\
\text { Recall }\end{array}$ & with session & without session \\
\hline 0.01 & 0.3820892 & 0.3029991 \\
\hline 0.02 & 0.3255506 & 0.1189198 \\
\hline 0.03 & 0.2960225 & 0.0283582 \\
\hline 0.04 & 0.1881605 & 0.0271492 \\
\hline 0.05 & 0.1744613 & 0.011988 \\
\hline 0.06 & 0.1708858 & 0.0082911 \\
\hline 0.07 & 0.1613805 & 0.0080912 \\
\hline
\end{tabular}

Table 2. Threshold Vs Average Recall

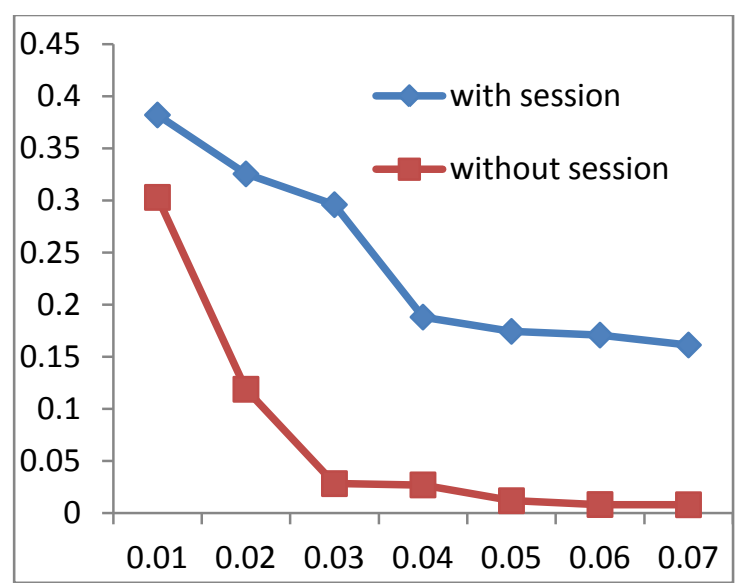

Fig.9 Threshold Vs Average Recall

\begin{tabular}{|l|l|l|}
\hline $\begin{array}{l}\text { User/ True } \\
\text { Positives }\end{array}$ & with session & without session \\
\hline $\mathrm{U}_{1}$ & 8 & 0 \\
\hline $\mathrm{U}_{5}$ & 2 & 0 \\
\hline $\mathrm{U}_{13}$ & 8 & 2 \\
\hline $\mathrm{U}_{20}$ & 3 & 2 \\
\hline $\mathrm{U}_{22}$ & 5 & 1 \\
\hline $\mathrm{U}_{24}$ & 101 & 80 \\
\hline $\mathrm{U}_{29}$ & 6 & 0 \\
\hline $\mathrm{U}_{38}$ & 0 & 0 \\
\hline $\mathrm{U}_{49}$ & 0 & 0 \\
\hline $\mathrm{U}_{50}$ & 45 & 0 \\
\hline
\end{tabular}

Table3. Users Vs no. of True Positives for Session

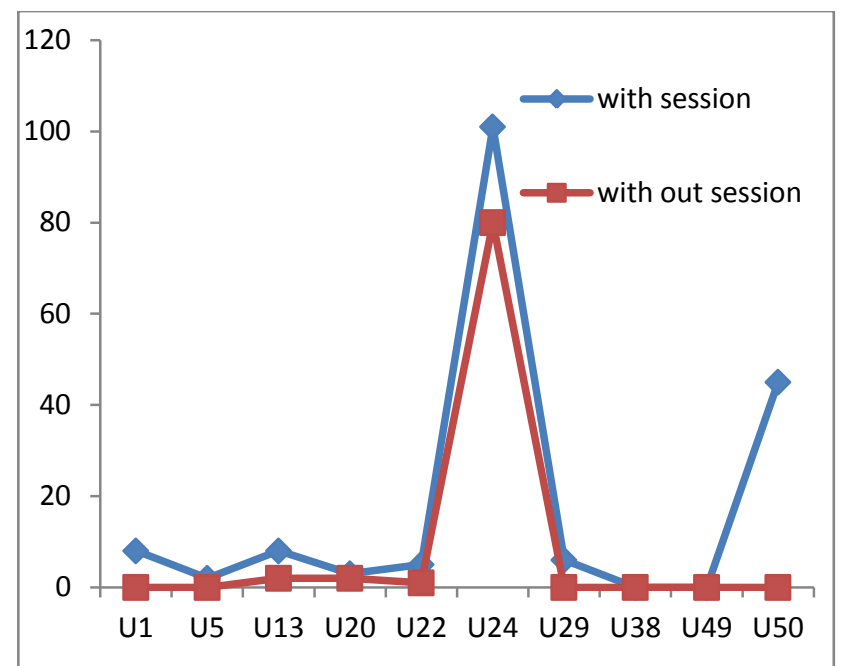

Fig. 10 Users Vs no. of True Positives for Session 4

\begin{tabular}{|l|l|l|l|}
\hline $\begin{array}{l}\text { Threshold/ } \\
\mathrm{P}, \mathrm{R}, \mathrm{F}\end{array}$ & $\begin{array}{l}\text { Precision } \\
(\mathrm{P})\end{array}$ & Recall (R) & $\begin{array}{l}\text { F-measure } \\
(\mathrm{F})\end{array}$ \\
\hline 0.01 & 0.0762619 & 0.7029991 & 0.1375972 \\
\hline 0.02 & 0.0850402 & 0.1189198 & 0.0991661 \\
\hline 0.03 & 0.0860341 & 0.0283582 & 0.0426563 \\
\hline 0.04 & 0.0795551 & 0.0271492 & 0.0404831 \\
\hline 0.05 & 0.0606562 & 0.011988 & 0.0200195 \\
\hline 0.06 & 0.0707849 & 0.0082911 & 0.0148436 \\
\hline 0.07 & 0.083704 & 0.0080912 & 0.014756 \\
\hline 0.08 & 0.1102565 & 0.0196916 & 0.0334153 \\
\hline 0.09 & 0.1394788 & 0.1519046 & 0.1454268 \\
\hline 0.1 & 0.1549326 & 0.1513282 & 0.1531092 \\
\hline
\end{tabular}

Table4. Precision ,Recall and F-measure for without session

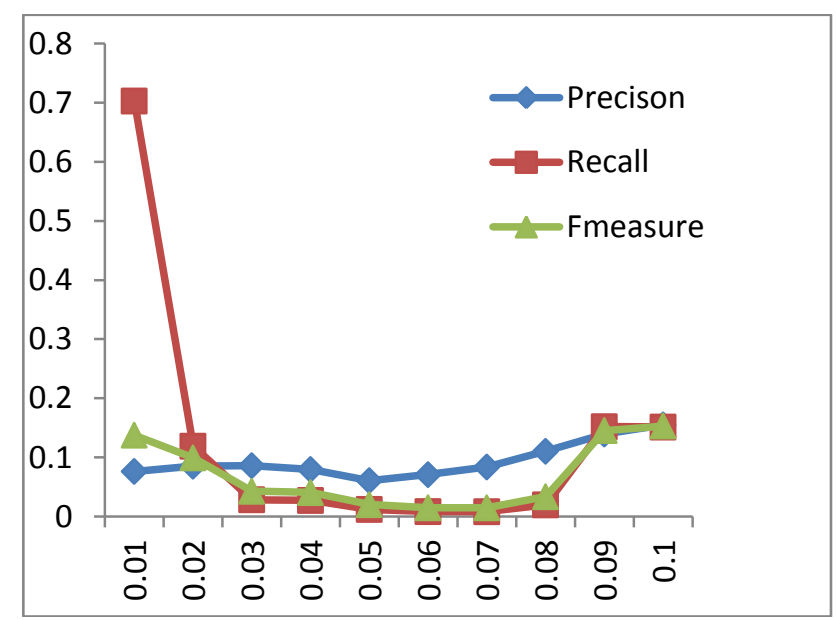

Fig.11 Precision, Recall and F-measure for without session 


\begin{tabular}{|l|l|l|l|}
\hline $\begin{array}{l}\text { Threshold/ } \\
\mathrm{P}, \mathrm{R}, \mathrm{F}\end{array}$ & Precision(P) & Recall (R) & $\begin{array}{l}\text { F-measure } \\
(\mathrm{F})\end{array}$ \\
\hline 0.01 & 0.0355047 & 0.3820892 & 0.064972 \\
\hline 0.02 & 0.0549396 & 0.3255506 & 0.0940136 \\
\hline 0.03 & 0.054887 & 0.2960225 & 0.0926039 \\
\hline 0.04 & 0.0973042 & 0.1881605 & 0.1282737 \\
\hline 0.05 & 0.086108 & 0.1744613 & 0.1153053 \\
\hline 0.06 & 0.1122663 & 0.1708858 & 0.1355082 \\
\hline 0.07 & 0.1123151 & 0.1613805 & 0.1324498 \\
\hline 0.08 & 0.0884416 & 0.06164 & 0.0726477 \\
\hline 0.09 & 0.0975431 & 0.060515 & 0.0746918 \\
\hline 0.1 & 0.1065505 & 0.081498 & 0.0923554 \\
\hline
\end{tabular}

Table 5. Precision, Recall and F-measure for with session

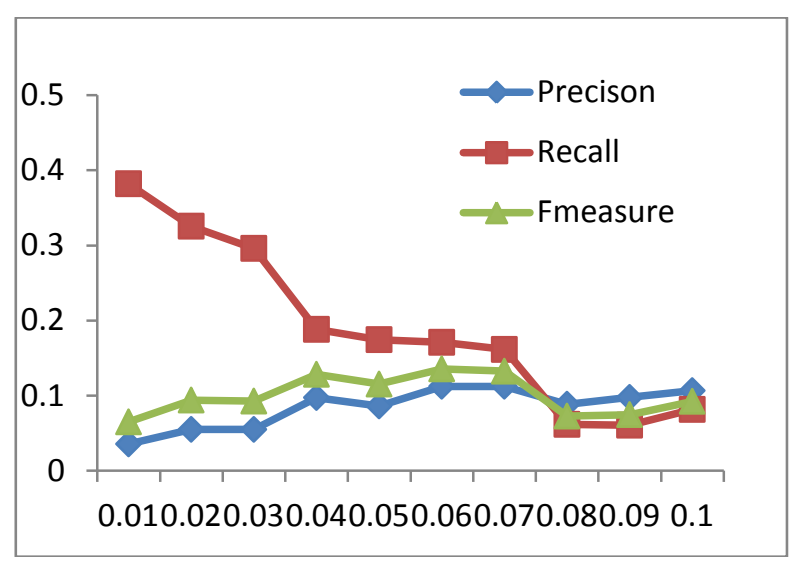

Fig. 12. Precision, Recall and F-measure for with session

\section{CONCLUSION AND FUTURE SCOPE}

We have discussed about the Session aware music recommendation system with user-based and item-based collaborative filtering method. The proposed algorithm takes the user interest into consideration without taking the user feedback explicitly as user logs are one of the implicit feedback. We also evaluated our system on benchmark dataset. We showed that session aware recommendation system improved traditional collaborative filtering technique. This work can be extended for recommendations to address Sparsity problem by taking dimensionality reduction techniques such as SVD or PCA into consideration.

\section{REFERENCES}

[1] Breese J, Hecherman D, Kadie C. Empirical analysis of predictive algorithms for collaborative filtering. In: Proceedings of the 14th Conference on Uncertainty in Artificial Intelligence (UAI'98). 1998. 43-52.

[2] Chong-Ben Huang, Song-Jie Gong, Employing rough set theory to alleviate the sparsity issue inrecommender system, In: Proceeding of the Seventh International Conference on Machine Learning and Cybernetics (ICMLC2008), IEEE Press, 2008, pp.1610-1614.

[3] Sarwar B, Karypis G, Konstan J, Riedl J. Item- Based collaborative filtering recommendation algorithms. In: Proceedings of the 10th International World Wide Web Conference. 2001. 285-295.

[4] Manos Papagelis, Dimitris Plexousakis, Qualitative analysis of user-based and item-based prediction algorithms for recommendation agents, Engineering Application of Artificial Intelligence 18 (2005) 781789.

[5] Hyung Jun Ahn, A new similarity measure for collaborative filtering to alleviate the new user cold- starting problem, Information Sciences 178 (2008) 37-51.

[6] SongJie Gong, The Collaborative Filtering Recommendation Based on Similar-Priority and Fuzzy Clustering, In: Proceeding of 2008 Workshop on Power Electronics and Intelligent Transportation System (PEITS2008), IEEE Computer Society Press, 2008, pp. 248-251.

[7] SongJie Gong, GuangHua Cheng, Mining User Interest Change for Improving Collaborative Filtering, In:Second International Symposium on Intelligent Information Technology Application(IITA2008), IEEE Computer Society Press, 2008, Volume3, pp.24-27.

[8] Duen-Ren Liu, Ya-Yueh Shih, Hybrid approaches to product recommendation based on customer lifetime value and purchase preferences, The Journal of Systems and Software 77 (2005) 181191.

[9] Million Song Dataset, official website by Thierry Bertin-Mahieux, available at: http://labrosa.ee.columbia.edu/millionsong/.

[10] M. Sunitha Reddy ,Dr. T. Adilakshmi, Music Recommendation System based on Matrix Factorization technique -SVD, International Conference on Computer Communications and Informatics (ICCCI14), Coimbatore, 3-5 January, 2014 